\title{
EI lenguaje visual y los mapas colaborativos para el desarrollo de ideas innovadoras
}

\author{
${ }^{a}$ Virginia Santamarina Campos, ${ }^{\mathrm{c}}$ María de Miguel Molina, ${ }^{\mathrm{d} B l a n c a}$ de Miguel Molinad, \\ ${ }^{\mathrm{b}}$ María Ángeles Carabal Montagud, ${ }^{\mathrm{e}}$ María del Val Segarra Oña y ${ }^{\mathrm{f}}$ Ángel Peiro Signes \\ a - bepartamento de Conservación y Restauración de Bienes Culturales; ${ }^{\mathrm{c}}$ - Departamento de \\ Organización de Empresas
}

\begin{abstract}
Cooperative learning, based on problem solving and the design of conceptual mobile maps, provides clarity and helps to make ideas more visible, tangible and sequential. Mobile cards allow to obtain system models more efficient by allowing more smoothly reiteration. On the other hand, teamwork with the use of mobile notes results in a richer systemic model, because it synthesizes different viewpoints, merging mental models and making it easier to understand and solve complex problems in a cooperative, creative and innovative way.
\end{abstract}

Keywords: cooperative learning, teaching innovation, teamwork, conceptual maps, mind maps, mental models, mobile cards, problem analysis, problem solving, project design

\begin{abstract}
Resumen
El aprendizaje cooperativo, basado en la resolución de problemas, mediante el diseño de mapas conceptuales móviles, proporciona claridad, ayudando a hacer las ideas visibles, tangibles y secuenciales. Las tarjetas móviles, permiten obtener modelos de sistemas más eficaces, al permitir la reiteración con mayor fluidez. Por otro lado el trabajo en grupo con el empleo de notas móviles da como resultado un modelo sistémico más rico, porque sintetiza diferentes puntos de vista, fusionando modelos mentales, y facilitando la tarea de entender y resolver problemas complejos de forma cooperativa, creativa e innovadora.
\end{abstract}

Palabras clave: aprendizaje cooperativo, innovación docente, trabajo en grupo, mapas conceptuales, mapas mentales, modelos mentales, tarjetas móviles, análisis de problemas, resolución de problemas, diseño de proyectos.

\section{Introducción}

El uso de notas móviles nos permite obtener modelos más eficientes y ricos para resolver problemas, ya que proporcionan una herramienta flexible para su análisis. Además, el diseño 
de mapas conceptuales móviles aporta claridad mostrando las ideas de una manera visual. Las secuencias del proceso pueden variarse, añadiendo ideas de una manera creativa, utilizando palabras clave o dibujos. Los objetivos y retos de cada sesión necesitan ser diseñados por anticipado para guiar a los alumnos durante el proceso de aprendizaje, independientemente de a qué área pertenezca la asignatura.

Por otro lado, se trabajan cuatro competencias transversales (UPV, 2017):

- Trabajo en equipo y liderazgo: "implica crear y desarrollar un clima de confianza mutua entre los componentes que permita trabajar de forma responsable y cooperativa...compartir conocimientos, compromiso y responsabilidad. Supone el reparto de tareas y roles y el respeto a las normas y reglas de juego establecidas por y para el grupo".

- Planificación y gestión del tiempo: "implica ser capaz de organizar y distribuir correctamente el tiempo del que disponemos y distribuirlo en función de las actividades necesarias para alcanzar nuestros objetivos a corto, medio y largo plazo".

- Innovación, creatividad y emprendimiento: "La innovación se entiende como la capacidad de dar respuesta satisfactoria a las necesidades personales, organizativas y sociales, modificando procesos y/o resultados para generar nuevo valor. A su vez, el desarrollo de esta competencia requiere, tanto el pensar de otro modo para aportar distintas perspectivas (creatividad), como el comprometer determinados recursos por iniciativa propia, con el fin de explorar una oportunidad, asumiendo el riesgo que esto comporta (emprendimiento)".

- Análisis y resolución de problemas: "Los problemas son situaciones nuevas que requieren que los individuos respondan con comportamientos nuevos. Resolver un problema implica realizar tareas que demandan procesos de razonamiento más o menos complejo y, en muchas ocasiones, no simplemente una acción asociativa y rutinaria".

El uso de mapas conceptuales, teniendo claros los objetivos y la manera de evaluarse, son una herramienta muy potente para afianzar los conceptos clave y conseguir un alineamiento constructivo (Biggs, 2004).

La metodologia se ha implementado en las siguientes asignaturas:

1. Product Development (31852). Obligatoria. Número de ECTS: 2,5. $\mathrm{N}^{\mathrm{a}}$ de estudiantes: 30. Facultad Administración y Dirección de Empresa, Master Universitario en Gestión de Empresas, Productos y Servicios.

2. Product Project (31855). Obligatoria. Número de ECTS: 20. N de estudiantes: 30. Facultad Administración y Dirección de Empresa, Master Universitario en Gestión de Empresas, Productos y Servicios.

3. Nuevas Tendencias en Estrategia de Servicio (31985). Optativa. Número de ECTS: 5. $\mathrm{N}^{\mathrm{a}}$ de estudiantes: 30. Facultad Administración y Dirección de Empres, Master Universitario en Gestión de Empresas, Productos y Servicios.

4. Metodologías de investigación (32257). Optativa. Número de ECTS: 3. $\mathrm{N}^{\mathrm{a}}$ de estudiantes: 30. Facultad Administración y Dirección de Empresa. Master Universitario en Gestión de Empresas, Productos y Servicios. 
5. Gestión del patrimonio inmaterial (33848). Optativa. Número de ECTS: 4,5. Na de estudiantes: 35. Departamento de Conservación y Restauración de Bienes Culturales, Máster Universitario en Conservación y Restauración de Bienes Culturales.

6. Desarrollo, gestión y dirección de proyectos de restauración. Optativa. Número de ECTS: 4,5. $\mathrm{N}^{\mathrm{a}}$ de estudiantes: 35. Departamento de Conservación y Restauración de Bienes Culturales, Máster Universitario en Conservación y Restauración de Bienes Culturales.

7. Historia de la Conservación y restauración de bienes culturales (33841). Optativa. Número de ECTS: 4. $\mathrm{N}^{\mathrm{a}}$ de estudiantes: 35. Departamento de Conservación y Restauración de Bienes Culturales, Máster Universitario en Conservación y Restauración de Bienes Culturales.

En el diseño de proyectos, la fase más importante es la de identificación, en la que se analizará la situación existente para crear una visión de la situación deseada y seleccionar las estrategias que se emplearán para lograrla. Hasta ahora una de las principales herramientas empleadas en el análisis de problemas para poder plantear una idea innovadora, ha sido el método dafo. Este método permite identificar los puntos fuertes y débiles de un área concreta y las amenazas y oportunidades del entorno, obteniendo una imagen global de la problemática del recurso patrimonial. A pesar de que esta técnica puede ayudar en la elaboración de estrategias o alternativas que mitiguen las debilidades a partir de las potencialidades y fortalezas existentes, no permite obtener una visión objetiva del problema focal, así como de sus causas y efectos. Actualmente la visualización de las relaciones causales de los problemas de una forma clara, supone una tarea fundamental. Por tanto la elaboración de un diagnostico que permita obtener un conocimiento profundo de la situación como base para la toma de decisiones, exige el empleo de una herramienta que permita entender porque se ha producido esa situación no desea y cuales con sus consecuencias, facilitando la priorización de los problemas y el llegar a un acuerdo colaborativo en el diagnóstico entre implicados.

En este caso los mapas conceptuales móviles cooperativos, han permitido visualizar las relaciones causales, priorizar los problemas y llegar a un acuerdo en el diagnóstico entre implicados, a través del trabajo colaborativo. La posibilidad de trabajar con mapas móviles, ha permitido la revisión colaborativa hasta verificar que el sistema es valido, haciendo los ajustes necesarios y llegando a consenso sobre su estructura.

\section{Objetivos}

El objetivo de la dinámica es analizar un problema complejo y buscar soluciones utilizando los mapas conceptuales móviles, de manera que a través de preguntas de enfoque el alumno vaya de lo general a lo específico, para buscar una solución en equipo.

De acuerdo con el "Modelo para la enseñanza del diseño creativo" de Glen et al. (2015: 190), podemos desarrollar distintas actividades:

1. Identificar problemas: a los equipos se les plantea un reto (o pregunta de enfoque) y deben identificar problemas y oportunidades.

2. Observación: pensando de la misma manera que el usuario. 
3. Visualización y reflexión: mostrando los resultados de la observación a toda la clase de manera visual para compartir, hacer preguntas y explicar.

4. Brainstorming de ideas, votando las mejores para reducir las soluciones.

5. Usar y probar prototipos, observando a los usuarios para captar nuevas ideas.

6. Test de viabilidad, explorando las opciones de modelos de negocio y las distintas posibilidades de generar valor.

En este caso, se han desarrollado actividades de los tipos 1, 3 y 4 . De todos modos, dependiendo de la asignatura o session se podrían añadir otras actividades. Por ejemplo, se podría hacer un juego de rol (role-playing) para aplicar las actividades 2 y/o 5. O hacer un estudio de caso utilizando la actividad 6.

\section{Descripción del desarrollo de la innovación}

Antes de comenzar la dinámica, son importantes dos tareas por parte del profesor:

- Redactar unas instrucciones previas para los estudiantes.

- Comprar el material necesario. Nosotros compramos tarjetas de la marca post-it que nos permiten manejar distintos tamaños, colores y formas.

Los grupos se forman con 3-5 alumnos. Se les plantea un reto y ellos deben identificar los distintos problemas, expresándolos con palabras clave o dibujos en notas móviles de un color determinado.

De acuerdo con el proceso de resolución de problemas, los grupos buscarán soluciones para cada problema por medio de la tormenta de ideas (brainstorming), de forma que al final se seleccionará una solución consensuada para cada problema. Dicha solución se expresará también con palabras clave o dibujos en una nota móvil de otro color, y que se conectará con el problema a resolver. Es decir, de cada problema una nota y de cada solución otra nota. En este momento, el professor revisará los modelos de cada grupo para detector información que falte por completer.

Al mostrar este modelo con las notas móviles pegadas en la pared, tendremos dos filas de ideas, problemas y soluciones. Entonces se plantea un nuevo reto, que es proponer medidas para prevenir que esos problemas aparezcan. Estas medidas, se escribirán o dibujarán en una nota móvil mayor y con un tercer color.

Asimismo, se podría plantear un tercer reto que sería establecer otras conexiones entre problemas y soluciones. Es decir, conectar soluciones que pueden ser communes para solucionar varios problemas.

Con el modelo final, el resto de grupos se desplazan por el aula para observar los modelos del resto de equipos y así pueden comparar dichos modelos con el suyo propio. Pero la mitad del grupo se queda en su modelo para explicar dudas a los "visitantes" o recoger las "recomendaciones" de sus compañeros. Acabada la ronda, cada grupo vuelve a reunirse para enriquecer su modelo con todas las ideas aportadas. 


\section{Resultados}

En ambas facultades, podemos comprobar que las ideas pueden representarse por texto (palabras clave) o con dibujos, dependiendo del reto propuesto y los objetivos.

Este tipo de ejercicios visuales, les ayuda a organizar ideas y a entender las relaciones o "conectores" entre los problemas y las soluciones. Además, las notas móviles permiten hacer cambios y resolver errores de una manera más sencilla, a la vez que los colores ayudan a diferenciar los conceptos.

La integración de distintos puntos de vista de modo colaborativo es muy importante en la resolución de problemas, por lo que el resultado final es más rico, pero, al mismo tiempo, es sencillo de comprender. Además, les ayuda a reflexionar sobre la necesidad de tener en cuenta el punto de vista de distintos actores.

A partir de la digitalización de las distintas fases de los trabajos de los alumnos, se ha realizado un análisis cuantitativo de los nodos, enlaces, uso de imágenes o uso de palabreabas claves, y los resultados obtenidos han sido:

$1^{\circ}$. E1 $90 \%$ de los diseños siempre presentan nodos y enlaces.

$2^{\circ}$. Los diseños que combinan enlaces y nodos, muestran un nivel de complejidad en función del número de nodos que suelen ir entre 6 y 8 . Los que utilizan menos nodos son más sencillos de entender, mientras que los que emplean más de 13 dan como resultado mapas complejos que dificultan su comprensión. Por lo que el número adecuado de nodos en un mapa, si se quiere mostrar algo de forma visual, debería estar entre 5 y 13 .

\section{Conclusiones}

Esta experiencia nos ha mostrado que el uso de notas móviles ayuda a impulsar el trabajo colaborativo en distintas asignaturas, se usen palabras clave o dibujos. Y, evidentemente, el trabajo colaborativo es fundamental a la hora de resolver problemas (Arquilla y Motta, 2011), ya que distintos actores pueden estar involucrados y las relaciones interpersonales son fundamentales (Muñoz González, 2014). El intercambio de ideas enriquece la visión de los problemas y la generación de posibles soluciones.

Es cierto que los cursos de postgrado suelen tener grupos pequeños, por lo que estas dinámicas son más sencillas de aplicar y además ayudan a impulsar el trabajo colaborativo, a la vez que ayudan a gestionar el tiempo puesto que cada fase tiene un tiempo máximo para que la dinámica pueda completarse en la sesión. Y estas son competencias transversales o "soft" que demandan los empleadores (Wu-Pong et al., 2013), más allá de conocimientos específicos. Incluso si los estudiantes no son expertos en la materia, y vienen de titulaciones distintas, este trabajo cooperativo les ayuda a trabajar en equipos interdisciplinares.

Se abren distintas preguntas a la hora de evaluar este trabajo, puesto que es difícil marcar elementos objetivos que ayuden al profesor, siendo más una evaluación por observación en el aula, combinada con el resultado final. Algunas sugerencias podrían ser: valorar la integración del equipo, valorar la cantidad y calidad de ideas expuestas (problemas 
detectados y/o soluciones propuestas), valorar el número de conexiones entre los problemas y soluciones, valorar la capacidad de síntesis (a través de palabras clave o dibujos), valorar la creatividad a la hora de exponer el mapa, valorar el ajuste al tiempo dado, etc. Como conclusiones finales podemos destacar tres puntos:

1. Los diferentes diseños, representan mapas conceptuales, que revelan puntos de vista diferentes. A pesar de no ser expertos en diseño de mapas conceptuales, logran de forma intuitiva dividir procesos complejos en tareas simples, organizándolo y sintetizarlo en un mapa.

2. La facilidad con la que se puede modificar un mapa, está vinculada con nuestro interés de mejorar el modelo. Así que un sistema con notas post-it no solo es más dinámico y flexible, sino que también genera más nodos que los dibujos realizados directamente sobre el papel.

3. Los mapas conceptuales colaborativos, logran integrar la diversidad de puntos de vista individuales de todos los alumnos. Estos nuevos sistemas contienen un elevado número de nodos, incluso nuevas ramificaciones y patrones, empleando diferentes colores de tarjetas, pero para los alumnos no resultan complejos porque participan en la construcción de estos modelos comunes.

\section{Referencias}

ARQUILLA, V. y MOTTA, R. (2011). "Student brain: tools and methods to support the students' creativity and to improve the learning and co-learning processes. The Polibrain case" En: INTED2011: 5th International Technology, Education and Development Conference, pp. 4651-4661.

BIGGS, J. (2004). Calidad del aprendizaje universitario. Madrid: Narcea.

BUZÁN, T. (1996). The Mind Map Book: How to Use Radiant Thinking to Maximize Your Brain's untapped potential. New York (USA): Plume.

FERRERO Y DE LOMA-OSORIO, G. Ed. (2009). Identificación y formulación de proyectos de cooperación para el desarrollo: gestión del ciclo del proyecto y enfoque del marco lógico. Valencia: Editorial UPV.

GLEN, R.; SUCIU, CH.; BAUGHN, C.CH. y ANSON, R. (2015). “Teaching design thinking in business schools" en The International Journal of Management Education, vol. 13, no. 2, pp. 182-192. <http://dx.doi.org/10.1016/j.ijme.2015.05.001> [consulta: 25 marzo de 2017].

J.M. Muñoz González, R. Serrano Rodríguez and V. Marín Díaz, El aprendizaje colaborativo y su desarrollo a través de mapas mentales. Una innovación educativa en la formación inicial docente, Educatio Siglo XXI, Vol. 32, 2015.

K.C. Laudon and J.P. Laudon (2016), Management information systems: managing the digital firm, Boston: Pearson, 14th ed. 
LAUDON, K.C. y LAUDON, J.P. (2016). Management information systems: managing the digital firm. Boston: Pearson.

MUÑOZ GONZÁLEZ, J.M.; SERRANO RODRÍGUEZ, R. y MARÍN DÍAZ, V. (2015). “El aprendizaje colaborativo y su desarrollo a través de mapas mentales. Una innovación educativa en la formación inicial docente" en Educatio Siglo XXI, Vol. 32.

R. Glen, Ch. Suciu, C.Ch. Baughn and R. Anson, "Teaching design thinking in business schools", The International Journal of Management Education, vol. 13, no. 2, pp. 182-192, 2015, doi: http://dx.doi.org/10.1016/j.ijme.2015.05.001.

S. Wu-Pong, et al., "The Future of the Pharmaceutical Sciences and Graduate Education: Recommendations from the AACP Graduate Education Special Interest Group", American Journal of Pharmaceutical Education, vol. 77, no. 4, 2013.

V. Arquilla and R. Motta, "Student brain: tools and methods to support the students' creativity and to improve the learning and co-learning processes - The Polibrain case", Inted2011: 5th International Technology, Education and Development Conference, pp. 46514661, 2011.

WUJEC, T (2013). Got a wicked problem? First, tell me how you make toast. TED Global 2013.

$<$ https://www.ted.com/talks/tom_wujec_got_a_wicked_problem_first_tell_me_how_you_ make_toast?language $=$ en $>$ [Consulta: 22 de marzo de 2016]. 